\title{
CONSUMO DE ÁLCOOLEM COMUNIDADES INDÍGENAS BRASILEIRAS: UMA REVISÃO LITERÁRIA
}

Fernanda Matos Fernandes Castelo Branco¹, Marcela Jussara Miwa², Divane de Vargas ${ }^{3}$

Objetivos: Identificar e sintetizar as evidências científicas sobre o consumo de álcool em comunidades indígenas no Brasil. Método: Trata-se de uma revisão integrativa da literatura, usando as bases de dados SciELO, BIREME e Web of Science com estudos nos anos de 2005 a 2015, em português, inglês ou espanhol e disponíveis na íntegra. Resultados: Foram selecionados 07 estudos para análise. $\mathrm{O}$ estreitamento das relações, pela facilidade de acesso às aldeias bem como pelos diversos motivos que os indígenas têm de ir até a cidade, fez com que as bebidas alcoólicas fossem inseridas nas comunidades indígenas, tornando assim um objeto de consumo de interação social dessa cultura que aos poucos vem perdendo as características próprias. Conclusão: As populações indígenas são vulneráveis ao consumo merecendo novas pesquisas, com a articulação dos serviços de saúde e profissionais de enfermagem, garantindo melhorias na qualidade de vida, promovendo bem-estar e prevenindo agravos de forma efetiva.

Descritores: população indígena; alcoolismo; transtornos induzidos por álcool.

\section{ALCOHOL CONSUMPTION IN BRAZILIAN INDIGENOUS COMMUNITIES: A LITERARY REVIEW}

Objectives: To identify and synthesize scientific evidence on alcohol consumption in indigenous communities in Brazil. Method: This is a integrative review of the literature, using the databases SciELO, BIREME and Web of Science with studies from 2005 to 2015, in Portuguese, English or Spanish and available in full. Results: We selected 07 studies for analysis. The narrowing of relations, due to the ease of access to the villages as well as the various reasons that the Indians have to go to the city, caused that the alcoholic drinks were inserted in the indigenous communities, thus making an object of consumption of social interaction of that culture that gradually they are losing their own characteristics. Conclusion: Indigenous populations are vulnerable to consumption, deserving new research, with the articulation of health services and nursing professionals, guaranteeing improvements in the quality of life, promoting well-being and effectively preventing diseases.

Descriptors: indigenous population; alcoholism; Alcohol-induced disorders.

\section{EL CONSUMO DE ALCOHOL EN LAS COMUNIDADES INDÍGENAS DE BRASIL: UNA REVISIÓN LITERARIA}

Objetivos: Identificar y sintetizar las evidencias científicas sobre el consumo de alcohol en comunidades indígenas en Brasil. Método: Se trata de una revisión integrativa de la literatura utilizando las bases de datos SciELO, BIREME y Web de los estudios de la ciencia en los años 2005 a 2015, en portugués. Inglés o Español y está disponible en su totalidad. Resultados: Fueron seleccionados 7 estudios para análisis. El estrechamiento de las relaciones, por la facilidad de acceso a las aldeas así como por los diversos motivos que los indígenas tienen que ir hasta la ciudad, hizo que las bebidas alcohólicas fueran insertadas en las comunidades indígenas, haciendo asi un objeto de consumo de interacción social de esa cultura que, a poco viene perdiendo las características propias. Conclusión: Las poblaciones indígenas son vulnerables al consumo merecen nuevas investigaciones, con la articulación de los servicios de salud y profesionales de enfermería, garantizando mejoras en la calidad de vida, promoviendo bienestar y previniendo agravios de forma efectiva.

Descriptores: población indígena; alcoholismo; trastornos inducidos por el alcohol. 


\section{INTRODUÇÃO}

O uso de bebidas alcóolicas merece destaque por terem sido importantes, apesar das variações, na vida humana. Na Antiguidade foi utilizado como meio de se livrar das enfermidades e das impurezas até como objeto e oferenda aos deuses, bem como forma de obter prazer e relaxamento(1). $O$ álcool não é único entre as drogas com papel de aliviar o controle social, entretanto é o mais divulgado e existe forte relação do consumo de bebidas de teor alcóolico com as festividades e celebrações, servindo como potencializador do alívio das tensões sociais sobre comportamentos, bem como permissor de experimentar situações potencialmente perigosas ${ }^{(2)}$.

Segundo a Organização Mundial de Saúde (OMS), o álcool é consumido por grande parte das pessoas na maioria dos países do mundo; a cada ano, cerca de dois bilhões de pessoas consomem bebida alcóolica, correspondendo a $40 \%$ da população mundial acima de 15 anos de idade ${ }^{(3)}$. No Brasil, segundo o II Levantamento Domiciliar sobre uso de drogas psicotrópicas no Brasil, realizado no ano de 2005 indicou que o número de brasileiros, com idades entre 12 e 65 anos, dependentes de bebidas alcoólicas foi de $12,3 \%$, o que corresponde à população de 5.799 .005 pessoas $^{(4)}$.

A luz desses dados pode-se evidenciar a problemática do consumo de substâncias psicoativas na população não indígena, entretanto, quando se trata dos povos indígenas, tal situação torna-se problemática e é agravada pelo processo de vulnerabilidade. O censo demográfico realizado em 2010 pelo Instituto Brasileiro de Geografia e Estatística (IBGE) revelou que existem 896 mil pessoas que se declararam ou se consideravam indígenas. Destes, 572 mil que correspondem a 63,8\%, vivem em área rural e 517 mil, pontuando 57,5\%, moram em Terras Indígenas oficialmente reconhecidas, portanto as comunidades indígenas representam $12,5 \%$ da população brasileira. ${ }^{(5)}$

Conforme o I Levantamento Nacional sobre padrões de consumo de álcool e outras drogas, realizadas entre as populações indígenas, no qual foram pesquisadas onze comunidades indigenas, de sete etnias diferentes correspondendo a 1.455 indígenas de ambos os sexos, moradores nas aldeias com faixa etária entre 18 e 64 anos foi constatado que a proporção dos bebedores é maior em homens $(52,7 \%)$ do que as mulheres $(24,6 \%)$. As mulheres apresentam maior proporção do uso abusivo e os homens da dependência. A distribuição por faixa de idade mostrou que as pessoas que mais consomem bebidas alcóolicas estão entre 18 a 34 anos correspondendo a $43 \%$. Em relação ao grau de dependência, prevalece à faixa etária dos 18 a $24 \operatorname{anos}^{(6)}$.
Assim, considerando a problemática dos padrões de consumo de álcool em comunidades indígenas e a carência de estudos na área, verificou-se a importância de fazer um levantamento sobre as evidências científicas acerca do consumo de álcool em comunidade indigenas no Brasil.

Almeja-se que a partir desses dados se possa contemplar uma maior visibilidade acerca da temática diante da escassez de estudos na área, bem como incentivar e contribuir com o desenvolvimento de outras pesquisas, o que proporcionaria o avanço de trabalhos científicos com as mais variadas etnias indígenas existentes, sendo esta a maior potencialidade do presente estudo.

Diante disso tudo se espera que os órgãos competentes para o assunto, juntamente com as equipes de saúde, dentre eles a equipe de enfermagem e as lideranças indígenas, deem maior visibilidade à área de saúde mental, com destaque para a problemática do uso de álcool e outras drogas, visando assim à tomada de medidas preventivas e de minimização dos danos causados por essas drogas e na busca por entender os fatores que contribuem para esse consumo, mesmo que na maioria das vezes ele ocorra em ocasiões pontuais, pois ainda nessas situações geram danos consideráveis à saúde do indígena, afetado diretamente, como também à própria aldeia na qual reside e mantém seus vínculos sociais e afetivos.

Assim o objetivo do estudo é Identificar e sintetizar as evidências científicas sobre o consumo de álcool em comunidades indígenas no Brasil

\section{METODOLOGIA}

Neste estudo, optou-se por realizar uma revisão integrativa da literatura que é um método de revisão mais amplo, pois permite incluir literatura teórica e empírica bem como estudos com diferentes abordagens metodológicas. Este método tem como principal finalidade reunir e sintetizar os estudos realizados sobre um determinado assunto, construindo uma conclusão a partir dos resultados evidenciados em cada estudo, mas que investiguem problemas idênticos ou similares. (7)

Nesse estudo, para elaborar esta questão foi utilizada a estratégia PICo "P"=população/problema de estudo, "I"=fenômeno de interesse, "Co"=contexto(8).

Tendo por base essa estratégia, nesta revisão integrativa, o acrônimo utilizado se deu da seguinte maneira: "P"=indígenas, "I"=consumo de álcool, "Co"=comunidades indígenas. Deste modo a questão norteadora foi definida: Quais as evidências disponiveis sobre o consumo de álcool em comunidades indígenas no Brasil?

Os critérios de inclusão para este estudo foram: estudos primários publicados em português, inglês ou espanhol, estudos recuperados nas bases de dados no 
período de janeiro de 2005 a dezembro de 2015 e que estivessem de acordo com a temática abordada, ou seja, consumo de álcool em comunidades indígenas no Brasil e disponiveis na íntegra. Os critérios de exclusão foram: estudos secundários (revisão de literatura, seja narrativa, integrativa ou sistemática), cartas, editoriais, comentários, monografias, dissertações e teses bem como estudos que abordem outros tipos de drogas.

Em relação à seleção bibliográfica, foram utilizadas as seguintes bases de dados: SciELO (Scientific Eletronic Library Online), e BIREME (Biblioteca Virtual em Saúde) e Web os Science. O levantamento das publicações foi realizado no periodo de dezembro de 2015 a fevereiro de 2016. Depois de selecionados os descritores que serviram de base para elaborar as estratégias de busca foram realizados os seguintes cruzamentos: SciELO (população indígena) AND (alcoólicos) OR (alcoolismo) OR (bebidas alcoólicas) OR (transtornos induzidos por álcool), BIREME (população indígena) $O R$ (indigenous people) $O R$ (health of indigenous peoples) AND (alcoólico) OR (alcoolismo) OR (bebidas alcoólicas) OR (transtornos induzidos por álcool) OR (alcoholism) OR (alcohol abuse) e Web of Science (indigenous people) OR (health of indigenous peoples) AND (alcohol abuse) OR (alcoholism) AND Brazil.

Ao total foram encontrados 1399 artigos, sendo excluídos 401, portanto dos 998 foram excluídos 963 pornão se relacionar com a temática e 18 pelo tipo de estudo, restando assim apenas 17, destes 8 não respondiam à questão norteadora e 2 eram duplicados, restando ao final 7 artigos.

\section{RESULTADOS}

A partir dos estudos obtidos pode-se perceber que - ano que obteve maior número de publicação acerca dessa temática foi o ano de 2012 apresentando 3 estudos, seguido do ano de 2007 com 2 estudos e anos de 2011 e 2014 apresentou 1 estudo.

Os periódicos em que foram publicados tais estudos sofreram modificações, mas sempre relacionados à área da psicologia, psiquiatria e enfermagem. Os autores que publicaram se repetem dentre esses estudos selecionados e publicados, confirmando assim número reduzido de profissionais interessados nessa área de estudo.

A análise realizada a partir dos dados apurados é relevante, pois evidenciam o número reduzido de publicações. Essa escassez de resultados encontrados nos permite repensar a construção do conhecimento na área e despertar o interesse para promoção do bem-estar e cuidado humanizado aos consumidores de álcool em comunidades indígenas, vislumbrando um campo de atuação em expansão que merece ser mais explorado.

\section{DISCUSSÃO}

A partir dos estudos selecionados evidenciou-se que o estudo ${ }^{(9)}$ aponta a necessidade dos indígenas de valorizar os aspectos culturais buscando manter a tradição sem perder a identidade cultural. Entretanto devido às condições não favoráveis, aliado a facilidade de entrada do álcool devido ao turismo e das relações sociais, o álcool se torna um bem de consumo acessivel e bastante utilizado nas comunidades indigenas acarretando danos irreversiveis como morte e doenças além de problemas familiares, psicológicos e sociais. Portanto, o consumo abusivo, casos de dependência pela proximidade com a cultura branca, descaracteriza assim os povos indígenas, pois o uso está desvinculado aos rituais tradicionais e algo pertencente ao cotidiano com uso/abuso das substâncias destiladas.

O uso de bebidas de baixo teor alcóolico é utilizado nos rituais tradicionais não podendo ser caracterizado como uma doença ou casos de dependência devido ao significado quimérico grupal. Todavia nos dias atuais houve a grande perda dos costumes culturais e uso de bebidas destiladas, tendo a cachaça como bebida de primeira escolha entre esse grupo indígena devido à facilidade de acesso as áreas litorâneas bem como pela proximidade com a cultura branca. Esse mesmo estudo mostra as consequências oriundas desse uso desregrado como as doenças sexualmente transmissiveis, gravidez precoce e problemas sociais. Algo que merece destaque nessa pesquisa é o valor atribuído pelos profissionais acerca dessa problemática, pois muitos ainda associam o consumo do álcool como um problema de causa universal e acabam esquecendo-se de contextualizar os aspectos sociais e culturais, pois mesmo ainda que tenha ocorrido toda essa mudança no hábito de consumo ainda e se deve refletir pautado no contexto vivenciado pensando na melhoria das condições de saúde dessa população. ${ }^{(10)}$

Em se tratando de aspectos culturais, estudo que utilizou o questionário padronizado (C-Cutdown, A-Annoyed, G-Guilty, E- Eye opener) como instrumento de rastreio conclui que este é inadequado, pois se trata de um procedimento universal utilizado para identificar dependência e necessitase entender as situações sociais, sendo essas condições necessárias para adequar as respostas dos entrevistados. $\mathrm{Na}$ população do Alto Rio Negro consideram-se "beber problema" as pessoas que apresentam comportamento violento, ou que são exemplos ruins aos filhos e para a comunidade, ou ainda aquelas que se esquecem dos seus atos após ingestão de substâncias alcóolicas bem como as pessoas que apresentam comprometimentos nas atividades laborais ${ }^{(11)}$.

Estudo realizado entre os povos indígenas Karitiana, corrobora com demais estudos descritos anteriormente,

10 I Enferm. Foco 2018; 9 (3): 8-12 
pois menciona que a convivência demasiada com os povos não índios favoreceu a entrada da bebida destilada entre os descendentes de colonizadores, tornando o álcool bastante acessivel e que as festas das aldeias se assemelham às festas das cidades, denominadas de "bailes brancos" permeadas de bebidas destiladas de cana com alto teor alcoólico, tal como, a pinga. Afirma ainda que os estudos têm se ocupado em descontruir o conceito de "alcoolismo" nas comunidades indígenas pelo simples fato de considerar incoerente a denominação psicopatológica que é feita na cultura dos brancos. Entretanto quando se aplica instrumentos já validados à avaliação do uso de bebidas com teor alcoólico se torna inviável nas populações indígenas, pois apenas se desenvolve técnicas relacionadas à problemática do álcool sem considerar o contexto cultural ${ }^{(12)}$.

Pesquisa feita entre os Potiguara evidenciou que o uso do álcool está estritamente relacionado à diversão, às festividades e aos rituais indigenas e destaca a cachaça como a bebida mais consumida devido à facilidade de acesso e pelo baixo valor de custo. Complementando esses dados, estudo que objetivou averiguar a situação de vida, saúde e doença dos Potiguaras, aldeados na Paraíba, constatou que $41,8 \%$ dos entrevistados tem alguém na família que faz uso de álcool e desse percentual 27,3\% afirmam que a bebida ocasiona algum dano à família, causando até morte, como foi encontrado em $5,5 \%$ dos resultados ${ }^{(13)}$

A luz desse contexto, as práticas de cuidado e medidas de controle ao combate do uso de álcool mostram as lideranças comunitárias e a influência da igreja como medidas protetivas ao consumo de bebidas alcóolicas no qual adotam a postura da "substituição da bebida alcoólica pela aceitação da palavra de Deus" e buscam costumes que a bebida não se compõe parte do processo diário. Assim se pode evidenciar mais um estudo que buscou um "cuidado" pautado no modelobiomédico desconsiderando os aspectos culturais. Deste modo, afirma que os profissionais de saúde devem encadear as ações com práticas indígenas de saúde além de compreender o modelo biomédico pautado no diálogo e sem esquecer o contexto sociocultural junto ao processo de alcoolização, pois caso contrário não se terá êxitos nas medidas adotadas ${ }^{(14)}$

Nessa vertente o uso diferenciado das bebidas com teor alcoólico é fruto de mudanças que ocorreram dentro das aldeias, tais como o contato com o homem branco, denominado de contato interétnico, o aumento da circulação de bebidas nas aldeias, mudanças no uso tradicional e de socialização e o advento das bebidas industrializadas. Hoje o uso de tais bebidas tem se tornado cada vez mais precoce além da substituição do caxiri pelos destilados tanto nas situações de consumo coletivo tido tradicional como na inclusão de datas festivas no calendário indígena pela influência da cultura branca(15).

Deste modo, o estreitamento das relações, pela facilidade de acesso às aldeias bem como pelos diversos motivos que os indígenas têm de ir até a cidade, fez com que a inserção das bebidas alcoólicas fosse apresentada e aprovada pelos índios tornando-se assim um objeto de consumo de interação social dessa cultura que aos poucos vem perdendo as características próprias.

Portanto, discutir a problemática do uso de álcool em comunidades indígenas é de suma importância para uma análise reflexiva além de considerar o contexto do consumo para traçar as medidas assistenciais ${ }^{(16)}$.

Assim os resultados desse estudo sugerem que novas pesquisas sejam realizadas e vinculadas a essa temática para uma maior produção do conhecimento e aproximação dos profissionais de saúde, em especial, os enfermeiros para que assim possamos além de refletir fazer a implementação de políticas públicas voltadas para as comunidades indígenas. Esse estudo apresenta como limitação, a escassez de estudos bem como a não generalização para demais comunidades indigenas de outros países, mas ao mesmo tempo mostra-se uma pesquisa valiosa no campo da saúde mental de populações específicas.

Portanto, as implicações deste estudo para a prática assistencial ocorrem no sentido de contribuir para que órgãos e entidades que tratam da tutela indigenista, governamentais ou não, possam elaborar estratégias e políticas voltadas para o aprimoramento da saúde dessa população vulnerável, especificamente no que se refere ao uso de substâncias psicoativas, algo que proporcionará melhoria na qualidade de vida indígena e otimização do convívio coletivo nas aldeias.

\section{CONCLUSÃO}

A partir dos resultados apresentados pode-se concluir que a produção bibliográfica sobre o consumo de álcool em comunidades indígenas é pouco representativa quando comparada com outras temáticas e especialidades, observando que não se pode pensar no uso do álcool pautado apenas no uso negativo, pois é notório que, apesar dos malefícios causados, ainda se tem a questão cultural que é fortemente relacionado aos padrões de consumo. Além de que existe uma gama de variedade dos contextos nos quais os indígenas fazem uso do álcool.

Destemodo, evidenciam-seestudosquebuscam descrever o "processo de alcoolização" diferenciando do "alcoolismo", pois estudos que seguem esta última vertente não analisam na íntegra o consumo de bebidas alcoólicas nessa população específica, desconsideram o contexto vivido e assim adotam medidas sem respostas eficazes. 
Assim as populações indígenas são vulneráveis ao consumo pelo processo histórico de colonização e devido a sua aculturação, merecendo uma reflexão sobre a necessidade de uma assistência holística com abordagem psicossocial e que surjam novas pesquisas com mais vigor que impactem mudanças entre os profissionais de saúde, tais como os enfermeiros, já que são fonte de apoio e juntamente com a articulação dos serviços de saúde garantam melhorias na qualidade de vida dos indígenas promovendo bem estar e prevenindo agravos de forma efetiva.

\section{Agradecimentos}

Agradecemos o financiamento da pesquisa a Secretaria Nacional de Políticas sobre Drogas (SENAD), do Ministério da Justiça e Cidadania e a Escola de Enfermagem de Ribeirão Preto da Universidade de São Paulo (USP)

\section{REFERÊNCIAS}

1. Seibel SD,Toscano Junior A.Dependência de drogas. In: Dependência de drogas. São Paulo:Atheneu;2001.

2. Dias LF. Consumo de bebidas alcóolicas entre os povos indigenas do Uaçá.In: Souza MLP. Processo de Alcoolização Indigena no Brasil: perspectivas plurais. Rio de Janeiro: Editora Fiocruz;2013.

3. Anderson P. Chisholm D, Fuhr DC. Effectiveness and costeffectiveness of policies and programmes to reduce the harm caused by alcohol. The Lancet [Internet], 2009 [cited 2016 Feb 10]:373(9682):2234-2246. Available from: http://www.who.int/choice/ publications/p_2009_CE_Alcohol_Lancet.pdf

4. II Levantamento domiciliar sobre o uso de drogas psicotrópicas no Brasil : estudo envolvendo as 108 maiores cidades do país : 2005 (2006) / E. A. Carlini (supervisão) [et. al.],São Paulo : CEBRID - Centro Brasileiro de Informação sobre Drogas Psicotrópicas: UNIFESP Universidade Federal de São Paulo.

5. Instituo Brasileiro de Geografia e Estatistica [Internet].(2010).Brasilia [cited 2016 Feb 20]. Disponivel em: http://www.ibge.gov.br/home/

6. Brasil. Presidência da República. Secretaria Nacional de Politicas sobre Drogas (SENAD). Relatório brasileiro sobre drogas; (org): Duarte P. Stempliuk VA, Barroso LP. Brasilia;2009.

7. Pompeo DA, Rossi LA, Galvão CM. Revisão integrativa: etapa inicial do processo de validação de diagnóstico de enfermagem. Acta Paul Enferm [Internet], 2009 [cited 2017 Jun 12]; 22(4):434-438. Available from: http://www.scielo.br/pdf/ape/v22n4/al4v22n4.pdf

8. Joanna Briggs Institute. Reviwer's Manual. Adelaide (Australia): Royal Adelaide Hospital, 2014. Disponivel em: http://joannabriggs.org/assets/ docs/sumari/reviewersmanual-2014.pdf. Acesso em: 20 jan 2016.

9. Melo JRFD, Maciel SC, Oliveira RCC, Silva AO. Implicações do uso do álcool na comunidade indigena Potiguara. Physis[Internet].
2011 [cited 2016 Feb 23];21(1);319-333. Available from: http://dx.doi. org/10.1590/S0103-73312011000100019

10. Maciel SC, Oliveira RDCC, Melo JRFD. Alcoolismo em Indigenas Potiguara: representações sociais dos profissionais de saúde. Psicol ciênc Prof [Internet], 2012 [cied 2016 Feb 8]; 32(1):98-111. Available from: http://www.redalyc.org/articulo.oa?id=282022731008

11. Souza MLP, Schweickardt JC, GarneloL. Oprocesso dealcoolização em populações indigenas do Alto Rio Negro e as limitações do CAGE como instrumento de screening para dependência ao álcool. Arch clin psychiatry (São Paulo, Impr.), 2007; 34(2): 90-96.

12. Vianna JJB, Cedaro JJ, OttAMT. Aspectos psicológicos na utilização de bebidas alcoólicas entre os Karitiana. Psicol \& Socied [Internet], 2012 [cited Feb 08]; 24(1):94-103. Available from: http://www.scielo.br/scielo. php?script=sci_arttext\&pid=SO102-71822012000100011

13. Oliveira RDCCD, Silva AO, Maciel SC, Melo JRFD. Situação de vida, saúde e doença da população indigena Potiguara.Rev Minei Enferm [Internet], 2012 [cited Feb 10]:16(1); 81-90. Available from: http://www. reme.org.br/artigo/detalhes/504

14. Ghiggi junior A, Langdon EJ. Reflections on intervention strategies with respect to the process of alcoholization and self-care practices among Kaingang indigenous people in Santa Catarina State, Brazil. Cad Saúde Pública [Internet],2014 Jun [cited 2015 Jan 29];30(6):12501258. Available from: http://dx.doi.org/10.1590/0102-311X00108613

15. Souza MLP, Garnelo L. When, how, and what to drink: alcoholism among Indian peoples in the Upper Rio Negro, Brazil. Cad Saude Publica [Internet], 2007 Jul [cited 2015 Abr 30]:23(7):1640-1648. Available from: http://dx.doi.org/10.1590/S0102-311X2007000700015

16. Branco FMFC, Vargas, D. Processo de alcoolização - reflexões de problemas relacionados ao consumo em comunidades indigenas. J Nurs UFPE on line [Internet], 2017 Feb [cited 2017 Jan 30]:11(2):680-685. 